\title{
La polmonite da Streptococcus pneumoniae in età pediatrica: aspetti clinici e microbiologici.
}

\author{
Marta Zambolin², Antonietta Cavallaro', Liviana Da Dalt², Barbara Andreola², \\ Marino Scarin', \\ 'Servizio di Microbiologia e Virologia dell'Ospedale di Padova \\ ${ }^{2}$ Dipartimento di Pediatria Università di Padova
}

Key words: Pneumonia, Streptococcus pneumoniae, children

Clinical and microbiological features of Streptococcus pneumoniae associated pulmonary disease in children

\section{SUMMARY}

Community-acquired pneumonia (CAP) is still a pediatric common illness that afflicts children troughout the world. Streptococcus pneumoniae is one of the most common and virulent pathogens so an early diagnosis is very important to do a right treatment. Our study has evaluated the role of symptoms, chest radiograph and microbiological test to determine a S.pneumoniae pneumonia. We conducted a prospective study from January to September 2005 including 44 children (I month- 15 years old) admitted to the Padova Pediatric ED suffering from pneumonia confirmed with a chest radiograph. From the obtained results the nasopharyngeal aspirate culture and the pneumococcal urine antigen are complementary and we can make a right diagnosis if we associate this test with symptoms and laboratory test.

\section{INTRODUZIONE}

La CAP (community acquired pneumonia) è una patologia di comune riscontro in età pediatrica $\mathrm{e}$ diffusa a livello mondiale. Nei bambini con età inferiore ai 5 anni, la fascia notoriamente più colpita, l'incidenza annuale di polmonite (in Europa e Nord America) è di circa 35-40 casi su 1000, mentre in quelli di età tra i 5 e i 14 anni, è di circa 11-16 casi su 10001. In Europa, annualmente, i casi di CAP in età pediatrica ammontano a circa 2,5 milioni. Nei paesi industrializzati dal 3\% al $18 \%$ dei ricoveri negli ospedali pediatrici è dovuto ad infezioni a carico delle basse vie aeree, in particolar modo polmoniti ${ }^{2}$.

E' perciò evidente come, nonostante la disponibilità di nuovi metodi diagnostici, di farmaci antimicrobici adeguati e specifici e di politiche sanitarie rivolte alla prevenzione, attualmente la polmonite in età pediatrica rappresenta una patologia notevolmente diffusa e potenzialmente grave ${ }^{3}$.

Tutto ciò rappresenta un impatto non trascurabile sul piano sociale ed economico, per il ricorso frequente a visite mediche e a terapie e conseguente riduzione della qualità di vita del paziente e perdita di giornate lavorative da parte dei genitori ${ }^{1}$.

Tra tutte le comuni patologie infettive a carico delle vie respiratorie la diagnosi microbiologica di polmonite è la più complessa in quanto l'isolamento dell' agente eziologico risulta particolarmente problematico ${ }^{4}$. Innanzitutto il prelievo di campioni respiratori delle basse vie nella popolazione pedia- trica è complicato e qualora effettuato, non sempre è qualitativamente rappresentativo della sede di infezione. La letteratura internazionale riporta che l'agente patogeno responsabile di polmonite viene identificato nel $24 \%-85 \%$ dei casi ${ }^{5}$.

Nella polmonite acquisita in comunità in età pediatrica $^{1,6}$ Streptococcus pneumoniae ha un ruolo principale ed è responsabile di gravi complicazioni; pertanto è essenziale giungere ad una diagnosi precoce per impostare una corretta terapia antimicrobica.

\section{Obiettivi}

Lo studio da noi intrapreso ha valutato il contributo della clinica, delle indagini radiologiche, microbiologiche e bioumorali al fine di giungere precocemente a una corretta diagnosi di polmonite da Streptococcus pneumoniae..

\section{MATERIALI E METODI}

Studio di tipo prospettico osservazionale condotto nel periodo gennaio - settembre 2005 su 44 bambini di età compresa tra 1 mese e 15 anni, giunti al Pronto Soccorso della Clinica Pediatrica di Padova per patologie respiratorie e con diagnosi clinica di polmonite confermata radiologicamente.

La raccolta dei dati anamnestici e l'esame clinico sono stati eseguiti al momento della visita in Pronto Soccorso. Su tutti i pazienti sono stati valutati i seguenti parametri: frequenza respirato- 
ria, saturazione di O2, temperatura cutanea ascellare. Tutti i bambini sono stati sottoposti alle seguenti indagini: ematochimiche (emocromo completo, PCR-reattiva); microbiologiche (esame colturale dell' aspirato nasofaringeo, ricerca dell'antigene pneumococcico nelle urine).

In tutti i bambini è stata eseguita una radiografia del torace in proiezione antero/posteriore per una eventuale diagnosi di polmonite interstiziale o addensamento parenchimale polmonare.

La polmonite complicata si associava a versamento pleurico, empiema o atelettasia.

L'aspirato nasofaringeo è stato eseguito inoculando $1 \mathrm{ml}$ di soluzione fisiologica sterile nelle cavità nasali e aspirando le secrezioni con un sondino collegato ad una siringa.

Il campione veniva seminato in agar cioccolato "PolyViteX" (bioMérieux) e in agar Trypcase Soy più $5 \%$ sangue di montone (bioMérieux ). Le piastre venivano incubate in atmosfera arricchita di $\mathrm{CO}_{2}$ al $10 \%$.

Nel caso di coltura positiva si è proceduto all'identificazione di colonie $\alpha$ emolitiche sospette per con le prove di sensibilità dell'optochina (Oxoid) e di solubilità in bile. Se le prove risultavano positive si allestiva l'antibiogramma per i seguenti antibiotici: Penicillina, Ampicillina, Eritromicina, Cefotaxime (Becton -Dickinson). La ricerca dell'antigene di Streptococcus pneumoniae nelle urine è stata effettuata tramite un test rapido basato su un principio immunocromatografico: Now-Streptococcus pneumoniae (Analitica Triveneta).

La ricerca dell'antigene nelle urine è un metodo recentemente approvato dalla US Food and Drug Administration. Non vi è consenso sul significato da attribuire alla positività del test, secondo alcu- ni autori è specifico e quindi utile per la diagnosi di CAP, secondo altri il test non può prescindere dall'indagine colturale di un campione delle basse vie perchè non permette di distinguere $\mathrm{i}$ pazienti con polmonite pneumococcica dai portatori dello Streptococcus pneumoniae nelle alte vie respiratorie $\mathrm{e}^{7,8,9,10}$

È stata utilizzata la regressione logistica univariata per tutte le coppie delle variabili prese in considerazione. Il confronto fra frequenze è stato eseguito mediante tabelle di contingenza, utilizzando il $\chi^{2}$ test. In caso di necessità, quando erano presenti degli zeri, è stato invece utilizzato il Fisher's exact test. Sono stati considerati significativi valori di $\mathrm{p}<0.05$.

\section{RISULTATI}

La popolazione esaminata era composta da 24 maschi $(54 \%)$ e 20 femmine (46\%); l'età media era pari a 3.7 , il $72.7 \%$ dei bambini aveva meno di cinque anni.

35 pazienti (80\%) erano vaccinati per Haemophilus influenzae, 5 (11\%) per Streptococcus pneumoniae, $4(9 \%)$ per Meningococco, 1 (2\%) per TBC, 42 $(95 \%)$ avevano eseguito la vaccinazione Pentavalente (difterite, tetano, pertosse, HBV, polio) e di 2 (4\%) non era noto lo stato vaccinale.

Solo 5 bambini (11\%) erano stati vaccinati verso lo pneumococco: 2 di questi (40\%) avevano l'esame colturale dell'aspirato naso-faringeo positivo e $2(40 \%)$ l'antigene urinario positivo.

Dei 39 bambini non vaccinati 11 (28\%) avevano la coltura positiva e 15 (34\%) l'antigene urinario positivo.

12 bambini (27\%) avevano iniziato una terapia antibiotica domiciliare: 6 con Penicilline o Amoxicillina, 4 con Macrolidi e 2 con Cefalosporine. 11 pazienti

Tabella I. Risultati dell' esame colturale e della ricerca dell'antigene pneumococcico

\begin{tabular}{lcccc}
\hline & ANF + & ANF - & Totale \\
\hline Ag. pneumococcico + & 8 & 9 & 17 \\
\hline Ag. pneumococcico - & 5 & 22 & 27 \\
\hline Totale & 13 & 31 & 44 \\
\hline
\end{tabular}

8 pazienti sono risultati positivi all'antigene nelle urine e all' esame colturale, 9 solo all'antigene, 5 solo alla coltura e 22 negativi ad entrambi i test.

Tabella 2. Relazione tra dati microbiologici, bioumorali e radiologici

\begin{tabular}{|c|c|c|c|c|c|c|}
\hline & Leucocitosi & $\begin{array}{c}\text { PCR> } \\
80 \mathrm{mg} / \mathrm{L}\end{array}$ & $\begin{array}{c}\mathbf{R x} \\
\text { addensamento }\end{array}$ & $\begin{array}{c}\mathbf{R x} \\
\text { interstiziale }\end{array}$ & $\begin{array}{c}\text { Versamento } \\
\text { pleurico }\end{array}$ & Totale \\
\hline $\mathrm{Ag+}$ & 4 & 5 & 7 & 1 & 2 & 8 \\
\hline Anf+ & $50 \%$ & $63 \%$ & $87 \%$ & $12 \%$ & $25 \%$ & \\
\hline $\mathrm{Ag}+$ & 9 & 6 & 8 & I & 4 & 9 \\
\hline Anf- & $100 \%$ & $67 \%$ & $87 \%$ & $11 \%$ & $44 \%$ & \\
\hline Ag- & 8 & 6 & 15 & 7 & 2 & 22 \\
\hline Anf- & $36 \%$ & $27 \%$ & $68 \%$ & $31 \%$ & $9 \%$ & \\
\hline Ag - & 3 & 4 & 3 & 2 & I & 5 \\
\hline Anf+ & $60 \%$ & $80 \%$ & $60 \%$ & $40 \%$ & $20 \%$ & \\
\hline
\end{tabular}

Degli 8 pazienti positivi all'antigene e all'esame colturale, 4 (50\%) presentavano leucocitosi, 5 (63\%) PCR-reattiva > 80 mg/L, 7

$(87 \%)$ un quadro di addensamento all'Rx torace, I (I2\%) un quadro interstiziale all'Rx torace, 2 (25\%) versamento pleurico. 
(25\%) avevano una temperatura cutanea inferiore a $38^{\circ} \mathrm{C}$ e 12 bambini (27\%) la saturazione dell'ossigeno era inferiore al $95 \%$.

Per quanto riguarda i sintomi clinici: 33 pazienti (75\%) presentavano tosse, $13(30 \%)$ impegno respiratorio $\left(\mathrm{FR}>95^{\circ} \%\right.$ ile per età e/o segni di dispnea), 5 (11\%) dolore toracico e in due casi è stato successivamente accertata la presenza di versamento pleurico.

In 32 bambini ( $73 \%$ ) erano presenti rumori patologici all'auscultazione.

Per 29 pazienti (66\%) è stato necessario il ricovero, 15 bambini (34\%) sono stati dimessi senza ulteriori accertamenti.

L'antigene pneumococcico è stato eseguito su tutti i pazienti: in $17(39 \%)$ è risultato positivo e negativo in $27(61 \%)$.

L'aspirato nasofaringeo è risultato positivo in 13 casi $(29,5 \%)$ e negativo in 31 casi $(70,5 \%)$.

8 pazienti sono risultati positivi all'antigene nelle urine e all' esame colturale, 9 solo all'antigene, 5 solo alla coltura e 22 negativi ad entrambi i test.

Degli 8 pazienti positivi all'antigene e all'esame colturale, $4(50 \%)$ presentavano leucocitosi, 5 (63\%) PCR-reattiva $>80 \mathrm{mg} / \mathrm{L}, 7$ (87\%) un quadro di addensamento all'Rx torace, $1(12 \%)$ un quadro interstiziale all' $R x$ torace, $2(25 \%)$ versamento pleurico.

Tutti i 9 pazienti con l'antigene positivo e l'aspirato nasofaringeo negativo presentavano leucocitosi, $6(67 \%)$ di questi con una PCR $>80 \mathrm{mg} / 1 ; 8$ $(87 \%)$ avevano un quadro di addensamento all'Rx del torace e $1(11 \%)$ con interstiziopatia, 4 (44\%) presentavano versamento pleurico.

Dei 22 pazienti con l'antigene negativo e l' aspirato nasofaringeo negativo $8(36 \%)$ avevano leucocitosi, $6(27 \%)$ PCR $>80 \mathrm{mg} / \mathrm{L}, 15(68 \%)$ addensamento all' $\mathrm{Rx}$ torace, 7 (31\%) interstiziale, $2(9 \%)$ con versamento pleurico.

Dei 5 pazienti con l'aspirato nasofaringeo positivo e l'antigene negativo $3(60 \%)$ presentavano leucocitosi e una PCR $>80 \mathrm{mg} / \mathrm{l} ; 2$ non avevano leucocitosi e di questi 1 con $\mathrm{PCR}>80 \mathrm{mg} / \mathrm{l} ; 3$ $(60 \%)$ avevano un quadro di addensamento all' $R x$ del torace e $2(40 \%)$ di interstiziopatia, $1(20 \%)$ versamento pleurico.

\section{DISCUSSIONE}

Dai nostri dati emerge un tasso di ricorso al Pronto Soccorso per broncopolmonite pari a circa 3 bambini ogni 1000 per anno, considerevolmente inferiore ai dati riportati in letteratura; una spiegazione può essere dovuta alla probabilità che un numeroso gruppo di bambini si rivolga al pediatra di base e non giunga alla nostra osservazione.

Quasi il 70\% dei bambini affetti da CAP, da noi osservati, è in età prescolare, con una incidenza in percentuale particolarmente elevata nei primi tre anni di vita, in linea con i dati della letteratura che sottolineano come l'incidenza di tale patologia, al di sotto dei 5 anni di età, sia la più elevata rispetto all'intera popolazione in età pediatrica.

Per quanto riguarda l'aspetto delle vaccinazioni non vi sono differenze significative tra il gruppo vaccinati ed il gruppo non vaccinati relativamente alla positività alle indagini microbiologiche ( $>00.05)$.

Non ci sono neppure differenze tra i due gruppi relativamente ai sintomi.

Come confermano i dati di letteratura, la presentazione clinica di tale patologia è aspecifica ${ }^{5,11,12} \mathrm{e}$ la triade classica: febbre, tosse, impegno respiratorio, si riscontra in una parte esigua di pazienti. Anche i valori della saturazione di ossigeno od eventuali sintomi di accompagnamento, quali dolore toracico e dolore addominale, non offrono un sostanziale contributo nel confermare il sospetto diagnostico $(p>0,05)$.

È quindi molto importante il ruolo delle indagini microbiologiche.

L'isolamento dello Streptococcus pneumoniae da campioni delle alte vie come l'aspirato nasofaringeo dimostra che il paziente è colonizzato e anche in presenza di CAP non può essere stabilita una associazione eziologica certa con la polmonite ${ }^{13,14}$. Solo campione di materiale delle basse vie ottenuto con tecniche invasive può consentire una diagnosi eziologia certa ma difficilmente applicabile nella popolazione pediatrica.

Invece la ricerca dell'antigene pneumococcio nelle urine sembra contribuire, in modo significativo, alla diagnosi di infezione delle basse vie respiratorie. Infatti, confrontando i pazienti con antigene positivo e aspirato nasofaringeo negativo con gli altri gruppi di pazienti, si è visto che il $100 \%$ presentava leucocitosi, il $67 \%$ una PCR $>80 \mathrm{mg} / \mathrm{l}$ e $1,89 \%$ un quadro radiologico di addensamento, segno di una polmonite più severa e quindi di una probabile eziologia pneumococcica.

E' importante precisare che la presenza di versamento pleurico e di empiema correlano con la positività dell'antigene nelle urine $(\mathrm{p}<0,05)$ indipendentemente dall'esame colturale e che fra $\mathrm{i}$ pazienti con antigene positivo e un quadro radiologico di interstiziopatia c'è una correlazione negativa significativa $(\mathrm{p}<0.05)$.

Dai nostri dati l'esame colturale dell'aspirato naso-faringeo, da solo, non è sufficiente per diagnosticare una polmonite da S.pneumoniae poiché si associa spesso ad un quadro ematochimico e radiologico variabile.

Va in più sottolineato che la raccolta del materiale dalle vie respiratorie superiori, anche se praticato di routine, spesso è difficile da eseguire sia 
per la scarsa collaborazione dei pazienti, sia per difficoltà operative.

Inoltre, il campione viene conservato in reparto e il tempo che intercorre tra il prelievo e la consegna al laboratorio può condizionare l'esito dell'esame colturale.

Streptococcus pneumoniae è un microrganismo che risente in modo rilevante degli stress ambientali e facilmente vi può essere una diminuzione della carica microbica iniziale. Tutto ciò associato al fatto che il prelievo deve essere eseguito nella fase iniziale prima della terapia antibiotica domiciliare o al momento del ricovero ospedaliero, in modo da non diminuire la sensibilità dell'esame colturale. L'esame colturale in ogni caso va sempre eseguito in quanto ci permette di eseguire l'antibiogramma essenziale sia a fini terapeutici che epidemiologici per il monitoraggio dell' antibiotico-resistenze.

In conclusione riteniamo sia necessario eseguire entrambe le indagini in quanto complementari. Tali indagini perché consentono di identificare, supportati dalla clinica e dagli esami ematochimici, uno degli agenti etiologici più importanti della CAP.

\section{BIBLIOGRAFIA}

1. Shoham Y, Dagan R, Givon-Lavi N, et al. Community- acquired pneumonia in children: quantifying the burden on patients and their families including decrease in quality of life. Pediatrics 2005; 115 : 1213-1219.

2. Juvén T, Mertsola J, Waris M, et al. Etiology of community-acquired pneumonia in 254 hospitalised children. Pediatr Infect Dis J 2000; 19: 293-298.

3. Sinaniotis CA. Viral pneumoniae in children: incidence and aetiology. Paediatric Respiratory Reviews 2004; 5(Suppl A): S197-S200.

4. McCracken GH. Diagnosis and management of pneumonia in children. Pediatr Infect Dis J 2000; 19: $924-$ 928.

5. Sinaniotis CA, Sinaniotis AC. Community-acquired pneumonia in children. Current Opinion in Pulmonary Medicine 2005; 11:218.

6. British Thoracic Society : BTS guidelines for the management of community acquired pneumonia in chilhood. Thorax 2002; 57: 1-24.

7. Esposito S, Bosis S, Colombo R, et al. Evaluation of rapid assay for detection of Streptococcus pneumoniae urinary antigen among infants and young children with possible invasive pneumococcal disease. Pediatr Infect Dis J 2004; 23: 365-367.

8. Dowell SF, Garman RL, Liu G, Levine OS, Yang YH. Evaluation of Binax NOW, an assay for detection of pneumococcal antigen in urine samples, performed among pediatric patients. Clin Infect Dis 2001; 32:824-825.

9. Neuman MI, Harper MB. Rapid antigen assay for the diagnosis of pneumococcal in children: a preliminary study. Ann Emerg Med 2002; 40:399-404.

10. Michelow IC, Lozano J, Olsen K, et al. Diagnosis of Streptococcus pneumoniae lower respiratory infection in hospitalized children by culture, polymerase chain reaction, serologic testing, and urinary antigen detection. Clin Infect Dis 2002; 34:e1-e11.

11. McIntosh K. Community-acquired pneumonia in children. The New England Journal of Medicine 2002; 346:429-437.

12. Ostapchuk M. Community-acquired pneumonia in infants and children. American Family Phisician 2004; 70: 899-908.

13. Heiskanen-Kosma T, Korppi M, et al. Etiology of chilhood pneumonia: serologic results of a prospecticve, population-based study. Pediatr Infect Dis J 1998; 17: 986-991.

14. Korppi M, Leinonen M. Pneumococcal immune complexes in the diagnosis of lower respiratory infections in children. Pediatr Infect Dis J 1998; 17: 992-995.
Marino Scarin

Via Giustiniani 2, 35100 Padova

Tel.: 0498213049 - Fax: 0498213054

E-mail: marino.scarin@sanita.padova.it 